\title{
Der Kantonsarzt - Bindeglied zwischen Medizin und Behörden
}

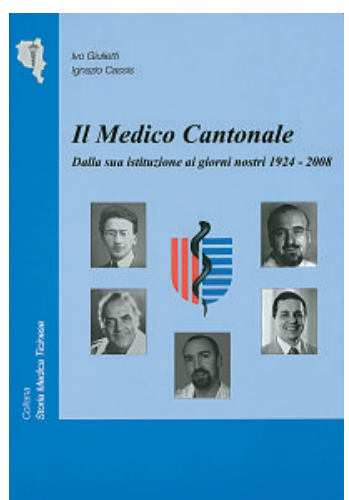

1 Giulietti I, Cassis I. Il Medico Cantonale - Dalla sua istituzione ai giorni nostri, 1924-2008. Herausgeber: Storia Medica Ticinese, Taverne. Zürich: Pro Litteris; 2009.

Das Buch kann beim Ufficio del medico cantonale bestellt werden: dss-umc@ti.ch

2 Martin J. Médecin cantonal - Pourquoi, comment pour quoi? Schweiz Ärztezeitung. 1986;67:1724-8.

3 Martin J. Quand il s'agit d'apprécier, d'arbitrer, de conseiller ... Expérience d'un médecin cantonal. Schweiz Ärztezeitung. 1998;79:480-3.

4 Martin J. Médecin et politique - Vie et société vues sous des angles différents. Schweizerische Ärztezeitung 2002;83(24):1243-8

* Jean Martin ist ehemalige Kantonsarzt im Kanton Waadt und Mitglied der SÄZ-Redaktion.
Den Lesern der Schweizerischen Ärztezeitung ist Ignazio Cassis ein Begriff. Cassis war von 1996 bis 2008 als hervorragender Kantonsarzt im Tessin tätig. Daneben hatte er von 2001 bis 2009 das Amt des Präsidenten der Schweizerischen Gesellschaft für Prävention und Gesundheitswesen (heute Public Health Schweiz) inne. Ein polyglotter, wie viele unserer italienischsprachigen Landsleute, wissenschaftlich kompetenter im Umgang stets freundlicher Kollege, der seit Juni 2007 auch als Nationalrat im Bundeshaus aktiv ist und ein Jahr später zusätzlich das Amt eines Vizepräsidenten der FMH übernommen hat.

Mit der grossen Unterstützung von Ivo Giulietti, Autodidakt der Medizingeschichte und Autor von sechs Bänden der Reihe «Storia Medica Ticinese», hat Ignazio Cassis in diesem Jahr das Buch «Il Medico Cantonale» [1], mit einem Vorwort des Leiters des BAG, Thomas Zeltner, veröffentlicht. Der gesamte erste Teil, der umfangreichste des Buches, ist eine Fundgrube spannender historischer Dokumente. Kapitel IV und V unter den Titeln «Die Arbeit des Kantonsarztes» und «Public Health» stammen aus der Feder von I. Cassis (der sich auch auf meine persönlichen Erfahrungen, vgl. [2,3] bezieht). Besondere Beachtung verdient der «Decalogo attitudinale per il Medico Cantonale»!

Die Aufgabe des Kantonsarztes bestand ursprünglich in der Bekämpfung von Infektionskrankheiten. Bis heute kommt ihr grosse Bedeutung zu, blickt man auf die Probleme unserer Zeit (saisonale Grippe und AH1N1, SARS, Masern, HIV/Aids, Hepatitis), doch hat sich das Aufgabenspektrum seither stark ausgeweitet. Eine wichtige Funktion des Amtes besteht in der Verbindung «Tra medicina e politica». Denn nahezu täglich muss der Kantonsarzt zwischen verschiedenen Seiten vermitteln: So erläutert er mal dem für Gesundheitsfragen zuständigen Regierungsrat, mal den Nichtmedizinern unter den Kollegen des Gesundheitsdienstes, mal Parlamentariern oder wiederum den Dienstchefs der kantonalen Stellen, vor welche Herausforderungen unsere Gesundheitsversorgung uns stellt. Und in der anderen Richtung kann der Kantonsarzt eine wichtige Rolle spielen, da er den Kontakt zu den praktizierenden Kollegen und der kantonalen Ärztegesellschaft hält.

Dabei tritt der Kantonsarzt selbstverständlich nicht als Botschafter der Ärzteschaft auf, der die Interessen des Berufsstandes gegenüber Behörden und Regierung vertreten würde. Obgleich er aus der Medizin kommt und mit der Arbeitsweise des Arztes vertraut ist, weshalb er die Aufgabe des Vermittlers übernehmen kann - sieht er sich im Dienste des Gemeinwohls. Es mag selbstgefällig erscheinen, doch lautete mein Ausspruch stets, «mein Patient ist die Bevölkerung unseres Kantons». In der Einzelkonsultation Patient-Arzt gilt der Grundsatz Salus aegroti suprema lex. Nun ist dieses höchste Gesetz aus der Sicht der öffentlichen Gesundheit in einem neuen Licht zu betrachten. Obgleich wir unseren Auftrag gegenüber dem Kranken weiterhin treu erfüllen wollen, müssen wir uns bewusst bleiben, dass es die Ressourcen der Gemeinschaft sinnvoll, fachgerecht und effizient zu nutzen gilt. In mehreren staatlichen Gebäuden ist bei uns der Grundsatz Salus publica suprema lex deutlich zu sehen (zu lesen auf den Seiten 150 und 206 des Buches). Es ist unter anderem Aufgabe des Kantonsarztes, eine harmonische Koexistenz, oder vielmehr eine gute Verbindung, zwischen diesen zwei verwandten Grundsätzen zu schaffen: der eine aus der Sicht des Therapeuten gegenüber seinem Patienten, der andere aus der Sicht der Regierenden gegenüber der Bevölkerung. Das Ziel von Arzt und Politiker scheint sich zu decken (Salus), aber ihr Patient/Klient, d.h. derjenige, der ihnen den Auftrag erteilt, ist in dem einen Fall eine Einzelperson, in dem anderen eine Gemeinschaft [4].

Es ist unmöglich, hier alle Aufgaben des Kantonsarztes aufzuführen. Überdies sind sie den praktizierenden Kollegen, zumindest in Teilen, bekannt. Man könnte sein Amt als das eines «Mädchens für alles», «Jack of all trades» beschreiben. Wichtig sind Glaubhaftigkeit, Überzeugungskraft und diplomatische Fähigkeiten. Er muss seine Positionen vertreten können und gleichzeitig Verhandlungsgeschick zeigen. Dabei sind ihm eine Portion strategisches und taktisches Denken von Nutzen. Und das Amt könnte den Weg zu weiteren Aufgaben ebnen - doch sind dies hier Gedankenspiele. Dem letzten Tessiner Bundesrat wurde nachgesagt, er habe «studiato da Consigliere federale sin dalla giovane età», von klein auf die Ausbildung zum Bundesrat begonnen, so gerade war sein Lebensweg auf dieses Ziel ausgerichtet. Dies gilt nicht für Ignazio Cassis. Er hat zunächst Medizin studiert und sich auf dem Gebiet Public Health spezialisiert. Indem er aber seine berufsethische Pflicht einer angemessenen Fortbildung ernst genommen hat, ist er in der Lage, Kompetenzen in anderen Bereichen zu erwerben. Wird er derjenige sein, der den FMH-Fähigkeitsausweis «Mitglied der Bundesregierung» ins Leben ruft?

Jean Martin* 